\title{
Movimiento social de Aysén. Un caso de análisis de incidencia ciudadana en la agenda de políticas públicas
}

\author{
Miguel Pérez \\ Universidad de Chile
}

\section{Resumen}

El presente estudio de caso tiene el objetivo de obtener aprendizajes para el diseño de políticas públicas con enfoque territorial. Para ello se propuso estudiar la movilización social de Aysén y su impacto en la agenda pública. La región de Aysén presenta un crecimiento económico estable, no obstante es la cuarta región más desigual del país. Ajustando regionalmente la línea de la pobreza acorde al costo de vida, ésta podría duplicarse en relación a la medición actual. Del total de los acuerdos planteados en la mesa social de Aysén, un $76 \%$ de las iniciativas emanadas del movimiento no fueron consideradas en el Plan de Gobierno 2010 - 2014. Por lo tanto, el nivel de incidencia en las políticas públicas fue bajo. La mayoría de las propuestas de gobierno se enfocaron en cambios de orientación de instrumentos públicos. En menor grado se generaron modificaciones a los métodos de gestión pública y definitivamente no se lograron cambios en la cultura organizacional del Estado. La resistencia a cambios profundos por parte del Ejecutivo queda en evidencia tras la omisión de acciones que pretendían impactar en el nivel más alto de incidencia en agenda pública.

Palabras clave: Conflictos sociales, movimientos sociales, Aysén, incidencia en agenda pública.

Aysen social movement. A case analysis of citizen impact on the public policy agenda

\begin{abstract}
This study was to learn lessons for the design of public policies with a territorial approach. We studied the Aysen social mobilization and its impact on the public agenda. The Aysen region has a stable economic growth, however it is the fourth most unequal region in the country. If we adjuste the poverty line according to the regional cost of living, it could be double from the current measurement. The $76 \%$ of the agreements taken in the Aysen social table, were not considered in the Government Plan 2010-2014. Therefore, the level of impact on public policies was low. Most government proposals focused on changes in orientation of public instruments. The modifications of governance methods were generated in lesser extent. Definitely the State organizational culture had no change. Resistance to deep changes by the Executive became evident after the omission of actions intended to change the highest level of impact on public agenda.
\end{abstract}

Keywords: Social conflicts, social movements, Aysen, influencing public agenda.

*Dirección de correspondencia [Correspondence

address]: Miguel Pérez, Universidad de Chile

E-mail: miguelperezbade@gmail.com 


\section{Introducción}

La emergencia de conflictos sociales ha generado en el último tiempo amplias discusiones a escala académica y política. En los últimos cuatro años se ha observado un incremento de las movilizaciones masivas de la sociedad civil, incidiendo de forma importante en la agenda pública. El caso de los estudiantes es uno de los más reconocidos, no obstante las comunidades regionales se han rebelado ante decisiones que afectan al porvenir de expectativas territoriales

El Movimiento Social de Aysén es uno de los eventos políticos y sociales más complejos ocurridos en los últimos años en Chile. Este se ha pretendido analizar desde diversos enfoques. Las principales miradas se han puesto en la manifestación y expresión política de una ciudadanía que transita desde un rol pasivo a un rol activo, siendo éste uno de los principales reflejos de este evento.

Nos enfrentamos a un conflicto político y social que emerge en Febrero del 2012, sin embargo el bagaje histórico que conlleva este movimiento se remonta por lo menos diez años atrás, donde se puede observar el nacimiento y desarrollo de una serie de actores políticos y sociales desde la ciudadanía.

En esta región, el Estado es muy relevante, dada la diversidad de mecanismos de política pública para el desarrollo de zonas extremas. Este jugó y sigue jugando un papel fundamental tanto en la resolución del conflicto como así también en la generación de nuevos mecanismos de acción pública.

Esta investigación buscó analizar la compleja relación que se produce cuando la ciudadanía intenta introducir cambios en la estructura del Estado altamente centralizado. El juego de poderes que se suscita en torno a este movimiento abre múltiples oportunidades de análisis. En primer lugar los cuestionamientos surgen en cuanto a la capacidad del Estado para responder a las necesidades de la ciudadanía a través de sus mecanismos de política pública.

El primer paso para penetrar en esta complejidad fue indagar sobre el tipo de políticas públicas que se han diseñado para el territorio de Aysén. Lo segundo fue reconocer la motivación de la ciudadanía que intentó incidir en el comportamiento del Estado. En tercer lugar observamos cómo estos dos mundos fueron capaces de acoplarse abriendo nuevas oportunidades para la configuración de una nueva agenda de políticas públicas.

Desde esta ventana de oportunidad tanto la ciudadanía como el Estado sufren cambios en su confi- guración basal post conflicto. Por una parte, el Estado incorpora aprendizajes acerca de la potencia de las emergencias sociales e intentan generar mecanismos para resolver las problemáticas planteadas por la comunidad. Por otro lado, la comunidad reconoce que logró organizarse para desestabilizar el equilibrio político, se empoderó y, si bien la evaluación colectiva no es del todo satisfactoria, el subconsciente comunitario aprendió una nueva forma de expresar sus demandas y propuestas sociales.

Lo interesante es reconocer qué tan profundos fueron estos cambios, y cuántos de ellos transitaron desde demandas sociales hacia instrumentos de política pública. Cualitativamente, se indagó si el problema público fue asumido como tal en la agenda de gobierno. La oportunidad que otorgó este conflicto social fue única para analizar en detalle procesos de configuración de agenda en las políticas públicas, la cual fue sometida a presiones de un entorno ciudadano empoderado.

Aún queda abierta la posibilidad de reconocer cuán potente fue esta agenda a la luz de los acontecimientos, cuántas propuestas fueron finalmente las que respondieron a una verdadera ventana de oportunidad en el diseño y ejecución de políticas públicas.

A la luz de estas premisas surgió la pregunta central: ¿Cuál fue el nivel de incidencia de la ciudadanía de Aysén en la agenda pública a partir del movimiento ciudadano "Tu Problema es mi problema"? ¿Cuáles fueron los mecanismos y/o procesos que sustentaron la movilización ciudadana en Aysén? ¿Cuál fue la respuesta del Estado chileno ante las demandas emanadas por el movimiento social tu problema es mi problema?

De esta manera, la presente investigación tiene como objetivo analizar el grado de incidencia del movimiento social "Aysén tu problema es mi problema" en la agenda pública del Gobierno Chileno para el territorio de Aysén.

\section{Modelo de corrientes múltiples}

Este trabajo está basado en el análisis de la formación de políticas públicas, que, al alero del modelo de corrientes múltiples de Kingdon (1984)Kingdon (1984), permite comprender cuán importante fue este movimiento para la configuración de una nueva agenda de gobierno desde el punto de vista territorial. Al mismo tiempo, se observa críticamente la interacción entre los actores que buscan ser protagonistas en la formación de la agenda política. Yáñez y Amigo (2009) citando a Kingdon (1984) definen que la agenda política: 
"es como la lista de temas o problemas que captan la atención de las personas dentro y alrededor del Gobierno en un momento determinado. La formación de la agenda es considerada como el proceso por el cual determinados problemas o cuestiones obtienen la atención seria y activa del gobierno como posibles asuntos de política pública (Ramírez, 2007). De esta manera, para John Kingdon (1984) el proceso de las políticas no se divide nítidamente en fases secuenciales, sino que se explica a partir de tres corrientes independientes que fluyen en el sistema, capaces de acoplarse cuando se abre una "ventana de oportunidad" haciendo posible el surgimiento de una política concreta como respuesta a un problema"

(Kingdon, 1984: 5)

Esencialmente la unidad de análisis del presente estudio de caso es la "agenda pública" que se configura a partir de la movilización social de Aysén. Las instancias de presión y movilización social, la configuración de la mesa de actores sociales de Aysén, la influencia de los medios de comunicación, los procesos de negociación, la interrelación entre los actores al interior y exterior del gobierno, serán analizados bajo la perspectiva de las corrientes para la formación de agenda.

El modelo de corrientes múltiples de Kingdon (1984) se compone de tres corrientes: de problemas, de soluciones y de políticas. Expuestas en los textos de Yáñez y Amigo (2009) citando a Ramírez (2007), podemos detallar que:

1. "Problems Stream" (Corriente de problemas): Para Kingdon los problemas no son solamente condiciones y acontecimientos externos, sino que además están constituidos por elementos perceptivos e interpretativos, por lo cual una condición pasa a convertirse en problema cuando para un determinado número de personas esta situación amerita la intervención pública, es decir cuando adquiere reconocimiento social. En la definición de qué es un problema, intervienen los valores (intereses de los actores y la concepción que cada uno tenga del estado ideal de las cosas), comparaciones (de la situación actual respecto a otros) y categorías (de los asuntos políticos que pueden determinar la definición de un problem

2. .

3. "Policy Stream" (Corriente de políticas, también conocido como corriente de solución): Consiste en el proceso de producción de ideas, que se asemeja al proceso de selección natural biológica, donde algunas ideas perduran, unas cambian, otras sobreviven y prosperan y el resto desaparece. Mientras que muchas ideas flotan en esta sopa primitiva de política (primeval soup), las que perduran son aquellas que superan el sistema natural de la selección.

4. "Politics Stream" (Corriente política): Está compuesta por el clima político, el "national mood", ambiente o clima político, que incide en la receptividad y acogida que pueda alcanzar una propuesta.

El autor nos indica que cada una de ellas es independiente y que en el plano ideal, la yuxtaposición de al menos dos de ellas permitiría generar la ventana de oportunidades donde se configuraría la agenda pública. Manteniendo esta estructura de análisis de políticas públicas, se revisa el contexto en el cual emerge la movilización de Aysén, intentando describir, a través de datos cuantitativos y cualitativos, el reconocimiento de las principales problemáticas del territorio.

Entenderemos las problemáticas en base a la línea teórica de Luis Aguilar (2007), quien destaca los aportes de Colber y Elder (1972 en Aguilar, 2007) acerca de la combinación del modelo integrado de agenda sistémica o pública desde la premisa de los conflictos de grupo. Estos logran hacerse visibles producto de tres requisitos:

"que sea objeto de atención amplia o al menos
de un amplio conocimiento del público, que una
buena parte del público considere que se requiere
algún tipo de acción y que a los ojos de los miem-
bros de la comunidad la acción sea competencia
de alguna entidad gubernamental"

(Colber y Elder 1972 en Aguilar, 2007: 34)

Para Kingdon (1984), la mixtura entre el problema y planteamiento de soluciones se constituye en una ventana de oportunidades, la cual se abre o cierra, en virtud de un momento político, desde donde emerge la decisión de la autoridad. Para Aguilar (2007), la formación de agenda y su proceso de toma de decisiones es realmente inestable, mala o laxamente estructurado, sin un patrón de comportamiento que mucho se asemeja a las anarquías organizadas que deciden según el modelo del Garbage can.

En esta lógica Aguilar (2007) rescata postulados de Kingdon (1984), quien introduce la idea de "policy windows": 
"Se trata de oportunidades favorables que permiten colocar determinados problemas y recomendar ciertas propuestas de solución. Sociedad y gobierno tienen que estar separados con diagnósticos y proyectos para cuando la dinámica política, de manera predecible o impredecible, configura una situación favorable para algún asunto en particular o para una familia de asuntos anteriormente menospreciados. Las ventanas no permanecen abiertas por mucho tiempo. Se cierran apenas los participantes perciben que el gobierno se ha hecho cargo de la cuestión, porque los participantes fallan al entrar en acción"

(Aguilar, 2007: 43)

Quién aproveche esta ventana de políticas, influirá en el planteamiento de las soluciones y del diseño de las propuestas de políticas. Aguilar (2007) lo plantea de manera muy cierta, señalando que quién define es quien decide. Aunque las fuerzas políticas logren aprovechar esta oportunidad, queda un importante trecho por recorrer para que ello finalice como parte de la agenda pública. Eso no quiere decir que los problemas tengan la solución esperada por los actores, pueden darse varios escenarios dignos de análisis, tales como definir problemas públicos mal estructurados.

Para Aguilar (2007) la relevancia de estructurar un problema permitirá reducir la incertidumbre e inestabilidad, no obstante señala que:

\begin{abstract}
"las políticas públicas son hipótesis de trabajo, no programas a seguir y ejecutar rígidamente (...) la política pública emerge, entonces como una tarea colectiva que incorpora conjunta y corresponsablemente la iniciativa social y la gubernamental, pues ante ciertos problemas se adolece teórica y tecnológicamente de una línea segura de respuesta. Por ende, como una estrategia susceptible de error y frustración (...) Por ello las condiciones necesarias son la democracia plural y competitiva que presiona a los gobiernos a hacer bien las cosas"
\end{abstract}

(Aguilar, 2007: 69-70)

Basados en este encadenamiento intencionado de categorías, se revisa el caso de la movilización social de Aysén, intentando concatenar los tres elementos emanados de los postulados de Kingdon, buscando comprender el surgimiento de la movilización en base a la corriente de problemas, reconociendo el origen de los problemas que impulsan el proceso de movilización social.

Desde el punto de vista de la corriente de soluciones, se indaga en las propuestas que surgen a los problemas presentados en el proceso de reivindicaciones sociales. Se analiza con detalle el nivel de empatía entre la agenda institucional y la nueva agenda emanada desde las bases sociales. Desde la vertiente de políticas, se comprenden los procesos de negociación, articulación y resolución que germinan en el aprovechamiento de la oportunidad para la configuración de una nueva agenda institucional.

\section{Metodología}

El presente proyecto de investigación utilizó una metodología de carácter cualitativo, basada en la técnica de estudios de caso investigativo, aplicando las herramientas de recopilación, análisis y evaluación de información de carácter primaria y secundaria.

En términos conceptuales la propuesta de estudio de caso investigativo nos permite transitar desde una mirada estática de los eventos y actores involucrados en la gestación y expresión del movimiento hacia una mirada procesual de éstos. Para ello se utilizará el método narrativo que nos permitirá explicar el flujo de acciones del movimiento recurriendo al contexto singular en el cual ellas ocurrieron. Finalmente de todo ello se obtiene un relato que nos permite conectar los acontecimientos, generando, organizando y analizando toda la evidencia empírica sobre el movimiento social de Aysén logrando explicar su desarrollo y principales aprendizajes.

Acorde a la idea de construir un estudio de caso investigativo definido:

\begin{abstract}
"como una pesquisa empírica en profundidad y multifacética qué: (1) investiga fenómenos contemporáneos en su contexto real (2); cuando los límites entre el fenómeno y el contexto no son evidentes o claramente diferenciables; (3) que utiliza diversas fuentes de evidencia o datos; (4) que utiliza primordialmente (pero no exclusivamente) métodos cualitativos de investigación"
\end{abstract}

(Orum, Feagin, Sjonberg 1991, Yin 1984, citados en Borgés (1995))

La investigación tuvo cuatro momentos de trabajo, los cuales a través de diversas fuentes fueron estructurando la información necesaria para contrastar las hipótesis planteadas.

1. Recopilación y Análisis de Fuentes Secundarias: A partir de la diversidad de fuentes secundarias generadas en torno al movimiento, 
se realizó una reconstrucción histórica del movimiento, incluso anterior al evento que se gatilla el 7 de febrero del 2012. Esta reconstrucción revisó diferentes fuentes bibliográficas tales como medios de prensa, revistas especializadas, opiniones de expertos, tanto escritas como audiovisuales. El rol que jugaron los medios de comunicación en la proliferación de este movimiento, nos desafió a recoger esta diversidad de información, filtrada a la luz de los acontecimientos más significativos.

En este primer proceso de revisión del estado del arte, se fue complementando el análisis de los discursos de los actores. Para ello se utilizó la matriz de análisis de actores e influencias en las diversas relaciones de poder. En primera instancia se identificaron los actores presentes en el contexto territorial, así también aquellos actores o instituciones que incidieron de una $\mathrm{u}$ otra forma en las dinámicas de relaciones de poder, y en las negociaciones del conflicto.

2. Entrevistas en profundidad: Se realizaron 16 entrevistas a diferentes actores involucrados en el proceso de movilización, negociación y resolución del conflicto: 2 Autoridades del Poder Legislativo; 1 Miembro del Consejo Regional; 2 Profesionales del Gobierno Regional; 1 Profesional experto en temas de regionalización; 1 Profesional experto en Identidad Regional; 4 Autoridades del Gabinete Regional; y 5 Dirigentes sociales participantes en la movilización y mesa social de Aysén.

3. Mesas de aprendizaje (focus group): De manera complementaria se generaron espacios presenciales de discusión con diversos actores sociales, expertos, observadores que manejan información respecto a procesos de incidencia de la ciudadanía en políticas públicas. A través de estas entrevistas grupales se fueron generando discusiones sobre la experiencia en Aysén. Discusiones desde el ámbito teórico y práctico que enriquecieron la información recopilada en los dos momentos previos.

4. Análisis de la Información: Para este proceso de análisis de los diversos discursos, se utilizó la técnica de análisis de contenido, revisando los principales argumentos relacionados con la temática estudiada, extrayendo la información más relevante y significativa.

\section{Corriente de problemas}

\section{Aislamiento Geográfico}

La sensación de aislamiento puede interpretarse desde el punto de vista de una conexión física con algo que se considera relevante, hasta la soledad o el abandono. La Subsecretaria de Desarrollo Regional (Subsecretaria de Desarrollo Regional, 2011a) define al territorio aislado en base a dos variables:

1. Aislamiento: Se refiere a un lugar (espacio físico) que se encuentra lejos, apartado, desconectado, con difícil acceso, incomunicado, o en situación de isla. En general se percibe como una condición "negativa". El aislamiento es determinado por factores físicos, de localización y demográficos y puede ser medido de forma que indique el "grado" o "nivel" de aislamiento de un territorio.

2. Integración: Es el conjunto de políticas, programas y proyectos que impulsa el Estado y en ocasiones los privados, para proveer servicios, conectividad y oportunidades de vínculo social, económico, político y cultural al lugar aislado (territorio) y su población para atender sus necesidades básicas de vivienda, salud, educación, trabajo, alimentación, vestuario, comunicación y en general todas aquellas necesidades que tiene la ciudadanía. La integración se mide en función de la oportunidad de acceder a los servicios públicos o privados. El diagnóstico regional realizado por la Universidad Central de Chile (2012), muestra que Aysén es la región más aislada a nivel nacional. El aislamiento estructural en promedio es de 0.51 , muy por encima del promedio nacional de 0.39 .

Tabla 1: Comparación índices de aislamiento comunal entre estudios SUBDERE 1999 2008

\begin{tabular}{|l|l|l|}
\hline COMUNAS & Ranking 1999 & Ranking 2008 \\
\hline Guaitecas & 1 & 51 \\
\hline Tortel & 2 & 19 \\
\hline O'Higgins & 3 & 18 \\
\hline Rí Ibáñez & 12 & 12 \\
\hline Lago Verde & 16 & 13 \\
\hline Chile Chico & 28 & 153 \\
\hline Cochrane & 29 & 111 \\
\hline Cisnes & 40 & 89 \\
\hline Aysén & 135 & 227 \\
\hline Coyhaique & 298 & 274 \\
\hline
\end{tabular}

Fuente: Elaboración propia en base a SUBDERE 1999 y 2008 
En la tabla 1 se observa la variabilidad de la posición comunal en el ranking nacional de aislamiento. Llama la atención que el mismo estudio del 2008, que coloca a Aysén en el último lugar de aislamiento regional, provoca cambios realmente radicales respecto a la posición de comunas tales como Guaitecas, Chile Chico, Cisnes y Aysén.

En la última medición realizada por la Subsecretaria de Desarrollo Regional (2011b) se puede observar la distinción de medición por localidades, lo cual agudiza el análisis respecto de la representatividad territorial. Según este estudio, en Aysén se encuentran 10.471 personas en situación de aislamiento, donde se destacan dos comunas con un $100 \%$ de aislamiento. Por el contrario, Coyhaique y Puerto Aysén tienen un nivel cercano al 1 y 7 por ciento, respectivamente.

No es factible generar comparaciones matemáticas, no obstante podemos relacionar que los nuevos indicadores están altamente influenciados por el criterio demográfico y el desarrollo urbano de las cabeceras comunales. En ambos casos estos criterios invisibilizan ciertas particularidades del litoral de Aysén, donde se ubican los principales asentamientos isleños de pescadores artesanales.

\section{Indicadores de pobreza y desigualdad}

Por décadas la región de Aysén ha representado el índice de pobreza más bajo a escala nacional. En el año 2011 la Encuesta CASEN señala que en Aysén la pobreza fue de $9,8 \%$ (en Chile fue de un $14,4 \%)$ y respecto a la pobreza extrema fue de un $1,6 \%$ (en Chile fue de un 2,8\%). Acorde a las comparaciones sostenidas entre el año 2009 y 2011, la región experimentó una de las bajadas más significativas en cuanto a la pobreza y pobreza extrema con un $35 \%$ y $69 \%$ respectivamente. Revisando los antecedentes expuestos por la Universidad Central de Chile (2012), se puede inferir que la razón principal de la caída porcentual de la pobreza se debe a la inyección de subsidios del Estado a las familias pobres.

En Aysén la pobreza aparece como una variable estadísticamente controlada. No obstante, acorde al mismo estudio citado anteriormente se puede constatar que mientras que el primer quintil alcanzó $\$ 166.851$, el quintil más rico llegó a $\$ 2.340 .171$, marcando una fuerte desigualdad de ingresos.

Las evidencias arrojadas por el índice de Gini son aún más contundentes. A medida que transcurren los años y el crecimiento de la población, sumado a nuevas oportunidades económicas, va aumentando la brecha de desigualdad salarial entre los sec- tores más ricos y más pobres. En Chile; a pesar de los bajos índices de desempleo, de crecimiento y reducción de la pobreza; la desigualdad se ha mantenido intacta. Para el caso de Aysén se aprecia una importante reducción en el año 2006, no obstante las siguientes mediciones del 2009 demuestran que Aysén fue más desigual que el promedio del país y en el año 2011, producto de los incentivos monetarios entregados a las familias pobres, esta brecha logra bajar un punto respecto del dato nacional.

Otro dato importante para la investigación se relaciona con el costo de vida en la región. Hay expertos del área indican que el costo de vida puede llegar a ser hasta un $30 \%$ más respecto del promedio nacional. No obstante, aparecen otros estudios que toman como referencia la ciudad de Coyhaique, e indican que comparativamente es una de las ciudades más baratas de Chile. Así lo indica el Informe "Índice de Costo de Vida de Ciudades 2010 (ICV)", realizado por el Centro de Investigación en Empresas y Negocios (CIEN) de la Facultad de Economía y Negocios de la Universidad del Desarrollo ubicando a Coyhaique en el lugar $\mathrm{N}^{\circ} 13$ de 14 ciudades estudiadas. Es importante señalar que el parámetro de costos estipulados es en base al nivel de gastos de un ejecutivo $\mathrm{ABC} 1$, que decida tomar la decisión de trasladarse de ciudad.

Por otro lado, el estudio realizado por el GORE señala un dato basado en la evaluación de los productos incluidos en el cálculo del IPC. Señala que: "El promedio simple para 98 productos incluidos en la estimación del IPC nacional entre enero y marzo del 2008, permite conjeturar que el promedio nacional del costo de la vida es $11,5 \%$ más alto en la ciudad de Coyhaique que en el resto de Chile" (GORE, 2009: 1932).

Asumiendo la brecha que existe entre los datos presentados anteriormente, tomaremos como referencia el estudio realizado por Valentina Ortiz (2013), quien en base al cálculo de la canasta básica de alimentos desarrollada por el Ministerio Desarrollo Social, impulsa un análisis comparativo del costo de vida en torno a las localidades que conforman la Cuenca del río Aysén, desde Balmaceda a Puerto Aysén, cubriendo el eje más poblado de la región.

El estudio realiza una detallada comparación de precios en base a productos genéricos de la canasta básica de alimentos en estas localidades, lo cual arroja finalmente una diferencia de $19,2 \%$ respecto del valor de la canasta básica de alimentos oficial del gobierno.

Este dato pone en entredicho los mecanismos de medición de pobreza a escala territorial, puesto que si se toma como referencia ese valor de cor- 
te estaríamos hablando que la pobreza aumentaría según Ortiz (2013) de 9,8\% a 15\% para el territorio regional. Especialmente para el caso de Coyhaique y Puerto Aysén aumentaría de $9,05 \%$ a $12.5 \%$ y $12,08 \%$ a $22.43 \%$ respectivamente.

\section{Crecimiento Económico}

Basados en datos oficiales, podemos evidenciar claros rasgos de crecimiento y sostenibilidad. En particular los datos macroeconómicos de Aysén, muestran una tendencia de estabilidad y crecimiento continuo. El documento de la Estrategia Regional de Desarrollo de Aysén (GORE Aysén) señala que: "Entre 1985 y 2006 la Región de Aysén experimentó un crecimiento promedio de 5,2\% anual, cifra ligeramente superior al promedio del país, que llegó a un 5,1\% promedio anual, lo que representa un dinamismo económico muy destacable" (2009: $53)$.

A partir del año 2000, Aysén experimenta su mayor crecimiento de los últimos 20 años, impulsados fuertemente por la reconversión del sector productivo acuícola, minería e industria. Considerando cifras más recientes extraídas desde el Banco Central (2012), se constata este nivel de crecimiento del PIB de forma estable a diferencia de lo que sucede en otras regiones del país.

A escala nacional la participación de Aysén lo ubica siempre en la última posición respecto de las demás regiones. En los puestos precedentes se ubican Arica y Parinacota, y Magallanes, que no logran incidir más allá de $1 \%$ en el PIB nacional.

Realizando la comparación entre estas regiones con menor cantidad de población, podemos observar que desde el 2010 a la fecha tanto AricaParinacota como Magallanes presentan una leve baja en su participación del PIB, por el contrario Aysén muestra una tendencia positiva desde el 2010 al 2012 .

La percepción de estabilidad macroeconómica de la región al año 2012, no auguraba procesos de malestar y crisis social. La región se destaca de sus pares, así lo establecen los indicadores más recientes. En su informe trimestral de enero a marzo del 2013, el INE señala que el Índice de Actividad Económica Regional (INACER) creció un 25,2\% respecto del mismo trimestre del 2012.

Cabe preguntarse por qué sobre este escenario de solidez macroeconómica podría haberse dado una manifestación social tan masiva. Para Valdebenito (2013) la cuestión se explica producto de la teoría de la Curva de J. Davies y el aumento de la priva- ción relativa, que se explica por

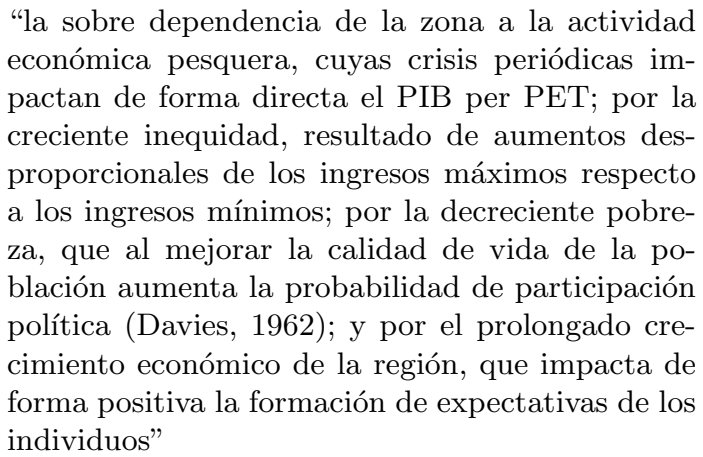

(Davies, 1962:3 en Valdebenito, 2012)

Las dimensiones planteadas por el autor son un tremendo avance para explicar cómo ciertos fenómenos macroeconómicos van generando expectativas en la comunidad, las cuales posteriormente son evaluadas socialmente. A nuestro juicio todo ello es parte del análisis, que debe ser complementado con otros elementos que la economía no logra dimensionar, tales como la capacidad organizativa de la comunidad, la identidad del territorio y las diferentes muestras de procesos de desarrollo social al interior de un territorio.

\section{Rol del Estado y actores sociales en Aysén}

El aparato institucional en Aysén es la pieza clave y angular de su proceso de desarrollo. Podemos apreciar en el gráfico $\mathrm{N}^{\circ} 3$ cómo la participación del sector público es la más importante a nivel regional, y a la vez observamos que desde el 2008 al 2012 mantiene un nivel de crecimiento positivo. El otro sector que asoma con relevancia regional es el de construcción, lo cual puede explicarse por el surgimiento de obras privadas asociadas a turismo, pero también es muy importante en esta materia las transferencias que hace el Estado para la construcción de obras viales.

En gran medida las decisiones asociadas a crecimiento, calidad de vida, incluso proyectos económicos, han estado asociados a la decisión del aparato público, especialmente ligadas al gobierno. Podríamos asegurar entonces que la dinámica de poder entre el gobierno y la comunidad se ha traducido principalmente en un Estado que resuelve los problemas del sector privado y la sociedad civil. Existen diversos instrumentos de planificación como políticas de desarrollo productivo para turismo, ganadería y ordenamiento territorial, entre otros. 
Sergio Boisier, 2004 plantea la labor del intendente regional como un animal con tres cabezas, en cuanto debe ejercer como gestor de políticas públicas a escala regional, como Presidente del Consejo Regional y como representante del Primer Mandatario. El autor nos muestra la compleja labor de gobernar a escala regional cuando existen atribuciones que en sí misma pudieran ser contradictorias.

Este animal de tres cabezas inconcebible para la realidad social, a nuestro juicio tiene al menos dos columnas: una que se ha creado al alero de las expectativas de una comunidad atenta a que su gobierno regional responda a la generación de expectativas fundadas en una especie de carta magna nominada Estrategia Regional de Desarrollo (EDR) al 2030 .

La otra columna es el Gobierno Nacional, el cual lleva doscientos años de ventaja institucional respecto al otro que aún se mueve en la esfera de lo imaginario. Cuando hablamos de lo subjetivo tiene que ver justamente con procesos que aún se sienten metafóricos y ajenos a la capacidad de concretar los sueños de largo plazo, con respecto a liderar la toma de decisiones desde las regiones.

Esta dicotomía es producto de los mismos mecanismos de modernización y traspaso del centro hacia las regiones, lo que para Lira Cossio (2003) es aún insuficiente para respaldar un ejercicio real de autonomía territorial, cuestión que es subyacente al modelo de desarrollo que hemos intentando impulsar como país.

La imagen objetivo de la ERD al 2030 representa la debilidad de un gobierno territorial que quiere ser autónomo y descentralizado, no obstante la tensión del centro hace que esta energía pase a ser por ahora un papel abstracto. La fuerza del movimiento social fue a nuestro juicio más allá de lo que pudo plasmar este documento de planificación regional, colocando en el debate temas a escala nacional que obedecen a la necesidad de un Estado más fuerte y con capacidad resolutiva a nivel regional.

Acorde al análisis realizado por el GORE (ERD, 2009), estudiando las redes de poder al interior de la región, podemos apreciar que los agentes que centralizan más poder fueron la Intendenta y el Consejo Regional (CORE). Evaluando el grado de salida (influencia), los agentes más importantes fueron el Consejo Regional, Secretaría Regional de Planificación y Cooperación (SERPLAC) y la Comisión Regional de Medio Ambiente (COREMA).

El estudio también analiza el tejido organizacional, en donde la sociedad civil muestra una baja tendencia a la participación social formal, comprendiendo esta como la adhesión formal a un organismo constituido legalmente.

La participación formal de la comunidad está asociada a la búsqueda de fondos concursables, en cambio lo que se ha generado en Aysén son movimientos en torno a demandas concretas. La comunidad de pescadores, por ejemplo, cada cierto tiempo se ha manifestado en contra de las medidas que afectan el desarrollo de su actividad productiva. En esta línea, observamos cómo los gremios de colectiveros o mujeres trabajadoras del Programa de Mejoramiento Urbano, se organizan para exigir subsidios o aumento de las cuotas de trabajo. Sumado a ello se ubican las organizaciones de empleados fiscales, los cuales en los últimos cuatro años han puesto en relevancia la exigencia de mejoras salariales.

En otra línea de organización podemos observar las organizaciones que se han manifestado abiertamente contra el modelo de desarrollo que pretende explotar a gran escala los recursos naturales de la región, las cuales se han conglomerado en torno a la idea de proteger a "Aysén como reserva de vida". Desde principios del 2000 surgen con fuerza organismos que se oponen a proyectos tales como ALUMYSA, instalación de salmoneras en el Lago General Carrera, la construcción de represas en la zona sur de Aysén.

Otros actores claves son los partidos políticos en Aysén. Estos gozan de una salud asociada a la estabilidad que arroja el sistema binominal. La configuración de coaliciones centro izquierda y derecha aún permiten que los principales cargos donde se ejerce poder político estén en manos de estos partidos. A partir del movimiento social esta estructura política sufre importantes modificaciones, considerando como hito relevante las primarias realizadas para elegir a un solo candidato entre los partidos comunistas, radical, demócrata cristiano, socialista y PPD. En dicha elección se impone un candidato no nombrado por las cúpulas partidarias, logrando el compromiso de todos los partidos en este camino electoral.

Sumando a ello la reaparición de Iván Fuentes como candidato a diputado, a través del cupo demócrata cristiano, la inscripción de Claudia Torres (Vocera Radio Santa María) como independiente, fueron evidencias concretas que la ciudadanía de Aysén no se moviliza tan sólo por efectos clientelares. Existe un trasfondo en la movilización de los actores de la sociedad civil, que sin duda el movimiento de Aysén logró plasmar y demostrar que la comunidad no estaba tan dormida como lo mencionaban las estadísticas formales de participación. 


\section{Inversión Regional: Programa Público de Inversión Regional (PROPIR)}

El Programa Público de Inversión Regional (PROPIR), es una herramienta de planificación regional que pretende realizar un seguimiento de las inversiones sectoriales y regionales. Actualmente este mecanismo de seguimiento está anclado a la página web www.chileindica.cl que permite mantener un monitoreo centralizado del nivel de concreción de las propuestas de inversión por cada servicio público.

La Dirección de Vialidad predomina por sobre los demás servicios. En segunda posición de relevancia se ubica el Gobierno Regional. Históricamente la inversión sectorial en la región de Aysén ha sido altamente superior, casi cuatro veces en promedio respecto de la inversión destinada a ser decidida por el ente regional.

Respecto de la misma gráfica podemos observar en posiciones secundarias a servicios involucrados en el desarrollo social de la comunidad, por ejemplo el Instituto de Previsión Social, que en tan sólo dos años de inversión logra ubicarse en cuarta posición. Recordemos que producto de la creación de la pensión solidaria los montos aumentan considerablemente, puesto que las pensiones son uno de los pilares de la reforma previsional.

Al realizar una análisis conglomerado de los aportes sectoriales versus regionales, podemos apreciar un crecimiento exponencial de ambas curvas de inversión. Ambos grupos presentan comportamientos similares de crecimiento, no obstante nuevamente la inversión sectorial crece 3 veces más en relación al primer año de medida. A diferencia de la inversión regional, que crece tan sólo 2.4 veces en relación al año 2007.

El $27 \%$ de los recursos totales de inversión regional del año 2012 son sancionados por autoridades regionales. En ello se cuenta todo el Fondo Nacional de Desarrollo Regional, Convenios de programación de la carretera austral y conexión marítima, Programa de Mejoramiento Urbano de Inversión Regional de Asignación Local (PMU IRAL), El Fondo de Solidaridad de Inversión social-Inversión Regional de Asignación Local (FOSIS-IRAL) e Inversión Regional de Asignación Local (ISAR) Agua Potable Rural.

Conglomerando datos de la inversión, volvemos a observar que desde el 2007 al 2012 el promedio de inversión en el área social emerge con gran fuerza acumulando un total de 50 mil millones anuales. Eso considerando solamente la inversión sectorial. En segundo lugar de relevancia se ubica la inversión en infraestructura, que ya analizamos anteriormente, donde el rol del Ministerio de Obras Públicas es altamente relevante.

Si efectivamente la inversión social es de alta importancia para el territorio, entonces qué hace pensar a la comunidad no sentir que este recurso esté llegando a resolver sus problemáticas, ¿Es un asunto de comunicación? ¿O más bien de definición de los énfasis de estas políticas públicas?

Si analizamos la posición relativa de Aysén respecto a indicadores de inversión, sorprende nuevamente la emergencia de la inversión social, en donde el porcentaje de inversión supera con creces a las otras áreas de desarrollo. Es importante mencionar que en esta área se conglomeran gastos en salud, educación, protección social, subsidios monetarios, entre otros.

Si bien no existe un parámetro exacto a escala nacional para comparar este dato, puesto que las estimaciones se realizan en base a la programación de gastos sectoriales y regionales, podemos tomar como referencia el Estudio de la División de Planificación Regional del Ministerio de Planificación (2010) donde indica que la Inversión pública efectiva per cápita país fue de $\$ 212.748$. El 2010 la inversión pública efectiva per cápita de Aysén fue de $\$ 744.757$, casi tres veces lo realmente invertido a nivel país. La brecha es muy amplia como para señalar que la región está ante una situación de abandono respecto de la capacidad del Estado para resolver problemáticas sociales.

La evidencia empírica grafica que los recursos existen, que la gestión del Estado está en marcha a través de sus herramientas de planificación, no obstante esto no tiene resonancia para sus comunidades.

\section{Conflictos en torno a la temática medio am- biental}

El Plan de Ordenamiento Territorial del Borde Costero de Aysén, diseñó una metodología para conciliar intereses de las comunidades y empresas inversionistas. Dicho diseño desemboca en la creación de una carta orientadora para la explotación de recursos y su implementación. Este instrumento es de carácter indicativo.

Nos referiremos a dos que a nuestro juicio tienen una relación causal con el surgimiento del movimiento social, y sus planteamientos de problemas sociales: el conflicto de la pesca artesanal y el rechazo al proyecto HIDROAYSEN

El conflicto de la pesca artesanal de Aysén se re- 
monta a principios del 2000, en torno a tres ejes centrales: 1) Discrepancias respecto a la preferencia de concesiones a la pesca industrial versus la reducción de cuotas al sector artesanal, 2) Discusión por zonas contiguas entre Aysén y Los Lagos, y 3) La baja fiscalización del Servicio Nacional de Pesca (SERNAPESCA) ante el ingreso de buques factorías a zonas destinadas a pesca artesanal.

Las organizaciones y gremios de pescadores artesanales asumen el rol de exigir demandas que están afectando a su sector y que ponen en jaque la sustentabilidad de su actividad, ante eso ejercen diversos mecanismos de presión que se observan desde el año 2003 a la fecha.

En la prensa escrita y sitios web proliferan los eventos en donde grupos de pescadores artesanales de Aysén se toman el Puente Aysén como medida de protesta para exigir sus demandas. El año 2009 luego de una rearticulación de las bases sociales, los pescadores vuelven a la lucha social, esta vez exigiendo mejores condiciones para el desarrollo y modernización de sus procesos de pesca.

El mundo de la pesca artesanal plantea la reivindicación de un modelo de desarrollo a escala humana, alejado de las grandes industrias, no descartando la modernización, pero si la industrialización de este rubro, batallando para sostener un estilo de vida basado en actividades sustentables para las futuras generaciones. La demanda contra el orden establecido está dada por la protección a la actividad ancestral y o a la saturación de grandes empresas y monopolios del sector pesquero. Sin duda que esta motivación ha llevado por años a levantarse contra el sistema que año a año los margina de su actividad, negándose a la esclavitud de puestos de trabajo precarios y poco rentables para garantizar la estabilidad de sus familias.

La otra fuerza que emerge en torno a la crisis de concentración del recurso hídrico en manos de la Empresa Endesa, en donde la sociedad civil se aglutina en torno al rechazo del megaproyecto hidroeléctrico "HIDROAYSÉN" en la zona sur de la región. Esto da pie a la generación de nuevas organizaciones con financiamiento local e internacional, las cuales están en contra de este modelo de desarrollo de crecimiento exógeno a los territorios. Durante años pequeños grupos de jóvenes, políticos y diversos organismos fueron dando lugar a un movimiento ciudadano.

Se fue creando una identidad común de rechazo a las formas de hacer desarrollo a costas de los recursos territoriales. Las experiencias nacionales de Ralco y otras dieron base para fortalecer la lucha contra este modelo industrializador. Lo interesante de este grupo de presión es que se configura a través de una gran gama de actores, tales como empresarios turísticos locales y extranjeros, inversionistas con fines de protección ambiental, grupos de jóvenes organizados y líderes regionales en el ámbito de la protección de los recursos naturales. El grupo de presión va adquiriendo características propias asociadas a tradiciones de los pioneros de la Patagonia Aysenina, muchos hijos de pioneros se comprometen con esta causa y son el principal renoval de esta corriente de ciudadanos que luchan por el medio ambiente, pero por sobre todo luchan por el respeto a sus tradiciones y a su territorio.

Por eso es clave el evento de la Cabalgata desde Cochrane a Coyhaique, en donde más de 300 jinetes a la usanza antigua recorrieron 300 kilómetros para expresar el rechazo ciudadano a este proyecto. Sólo en este tema se pueden observar cientos de redes sociales conectadas a lo largo del país difundiendo este evento, que marcó un antes y un después en la resolución de este conflicto de intereses ambientales.

Sin duda que la sinergia provocada en la región desde años anteriores desemboca en esta caudal de sociedad civil, ciudadanos cada vez más conscientes de su realidad, más compenetrados socialmente, se reúnen en esta confluencia de fuerzas políticas, fuerzas de poder local que dan pie al nuevo movimiento social Aysén tu problema es mi problema.

Estas dos corrientes de fuerzas locales, liderazgos comunitarios y luchas sectoriales toman mayor relevancia cuando emerge la manifestación del 7 de febrero del 2012 con la quema del bote frente al Puente Ibáñez. Ese día comienza a configurarse un nuevo escenario regional.

En segundo plano quedaron planteadas las demandas de la pesca artesanal, del movimiento Patagonia Sin Represas y otros grupos. En gran medida la lucha por los grandes temas llevó a los líderes de este movimiento a tener un gran respaldo de la ciudadanía, apoyando mayoritariamente las diversas acciones de presión, las cuales muchas veces colocaron en jaque la seguridad y estabilidad de la propia ciudadanía.

Es imposible dejar de destacar la bandera negra colocada en la mayoría de los hogares de Puerto Aysén y Coyhaique, lo cual representó el repudio a las acciones de fuerzas especiales en contra de los manifestantes y fortaleció la identidad colectiva de los habitantes de Aysén en torno a este movimiento. Asimismo se observaron grandes expresiones solidarias, como ollas comunes, cacerolazos y llamativamente fogones comunitarios en vez de barricadas nocturnas ${ }^{1}$.

\footnotetext{
${ }^{1}$ Cinco ollas comunes en Aysén alimentan
} 


\section{Conformación de la agenda (Corriente de soluciones)}

\section{Levantamiento de la agenda social (Movimiento Social)}

A partir del análisis de contenido, observamos que los discursos del Movimiento social hacen énfasis en la obtención de recursos; sin embargo, de forma subyacente, emergen códigos sustentados en la búsqueda de autonomía territorial. Se aprecia una mixtura entre una desazón basada en la exclusión, que exige integración, y una demanda de autonomía y poder territorial. Es decir, el elemento central que coloca el movimiento social en la mesa, es el poder y la autonomía que tiene la comunidad aysenina para rebelarse ante la administración concentrada en el Estado. La movilización se sustenta en la forma de hacer política pública, el que para los actores sociales, más allá de prestar soporte para el desarrollo de sus expectativas, ha centrado su accionar en la proliferación de intereses ajenos al territorio. Esto tiene consonancia con los planteamientos de Zibechi (2003), que identifica que los nuevos movimientos sociales latinoamericanos tienen un patrón en la organización del espacio geográfico.

Recurriendo a los postulados de Sidney Tarrow (1998) podemos definir a los movimientos sociales desde una lógica de las oportunidades que éstos pueden generar en el espectro político, las cuales no necesariamente son de carácter positivo respecto al levantamiento de sus demandas. Para el autor para que una acción colectiva se transforme en un movimiento social, debe contar con al menos tres recursos: "el repertorio de la acción colectiva que la gente emplea para conseguir apoyo e imponer su voluntad ante los oponentes, los marcos de dicha acción, que dignifican y justifican al movimiento y las estructuras de movilización de acción que refuerzan la primera línea que vinculan al centro con la base del movimiento" (Tarrow, 1998: 134).

Tito Tricot (2011) argumenta que la ocurrencia de movimientos sociales surge desde la tensión por el poder y la dominación en un determinado espacio y sociedad. Tomando en consideración una diver-

a manifestantes. http://www.soychile.cl/PuertoMontt/Sociedad/2012/02/24/73958/Cinco-ollas-comunesen-Aysen-alimentan-a-manifestantes.aspx (Consultado el 15 de enero de 2014)

Bandera negras en Aysén. http://www.elciudadano.cl/2012/02/25/48796/banderasnegras-en-aysen/ (Consultado el 15 de enero de 2014)

$\begin{array}{cccc}\text { Manifestantes } & \text { condimentan } & \text { con } & \text { hu- } \\ \text { mor las } & \text { barricadas } & \text { en } & \text { Aysén. }\end{array}$

http://www.biobiochile.cl/2012/02/28/manifestanteentrega-su-particular-humor-en-barricadas-deaysen.shtml (Consultado el 15 de enero de 2014) sidad de posturas (Gamson, 1992; Snow, Benford, 1992; Zald, 1999; Gamson Y Meyer, 1999), señala que los movimientos sociales parecen surgir cuando la sociedad se enfrenta, o al menos a sectores de ésta, a situaciones conflictuales irresolutas que requieren de nuevos sujetos con la capacidad de interpelación al poder. Es decir, poseerían un carácter al menos potencialmente antisistémico (Tricot, 2011).

Se suma a ello los postulados del regionalismo, el cual surge como una corriente fundada en el reconocimiento a las identidades territoriales. La lucha de Aysén se basa en el reconocimiento de las desigualdades interregionales por parte de sus ciudadanos (Abalos, 1985).

De esta manera, el Movimiento de Aysén se concibe bajo la lógica de un movimiento social con identidad territorial, basado en su trayectoria histórica, capaz de quebrar el status quo y colocar en el debate los temas estructurales que a la gente de Aysén le importan. Este movimiento visibilizó de manera estratégica las demandas de poder y autonomía del territorio aysenino, utilizando estrategias de negociación que permitieron colocar nuevos problemas públicos en la agenda de gobierno. Estos problemas públicos se plasmaron en la carta principal de navegación denominada "Demandas Movimiento Social por la Región de Aysén". Este documento adquiere sentido producto de la fuerza de sus actores sociales que se traduce en la creación de la ventana de oportunidad política, provocada por la fuerza del movimiento social, que fue capaz de colocar en la mesa de discusión de políticas públicas este documento.

\section{Análisis de las propuestas y su impac- to en la agenda pública}

La connotación pública que adquirió el movimiento social de Aysén dejó en evidencia la oportunidad política de avanzar en una agenda social para el desarrollo regional de Aysén. Así fue comprendido por los y las dirigentes de la mesa social. La evolución del conflicto fue avanzando con claridad en la instalación de problemas públicos, liderados por dirigentes sociales con amplia capacidad de convocatoria y liderazgo.

El punto de inflexión para aprovechar esta ventana de oportunidad se produce luego del intenso desgaste de las relaciones políticas, provocado por los actos represivos de Carabineros de Chile y el incremento de la fuerza del movimiento social a escala regional y nacional. La lectura política de este momento se vislumbra en la presencia de personeros públicos provenientes de la Secretaría General de la Presidencia que desde Aysén abren la posibi- 
lidad de diálogo con el Vicepresidente en la ciudad de Santiago. Este momento plasma la conexión casi perfecta del aprovechamiento político, donde los dirigentes de la mesa social en pleno asisten a una reunión en el Palacio de la Moneda, sosteniendo la reunión que pone fin a los bloqueos en la región de Aysén y da paso a los procesos de negociación formales entre la mesa social y el gobierno. A partir de este momento se establecen nuevos escenarios para la colocación de los nuevos temas de agenda pública.

A partir del análisis de fuentes secundarias y primarias, hemos reconstruido el proceso a través de un plano general de los once puntos vistos a la luz de los mecanismos de negociación, donde se constituyeron 8 mesas de negociación con sus respectivos acuerdos: Mesa de Vivienda Patagónica, Mesa de Educación Patagónica, Mesa de Pesca Artesanal, Mesa Campesina Patagónica, Mesa de Salud, Mesa de Infraestructura y Servicios Básicos (SUBDERE), Mesa de Transportes y Mesa de Participación Ciudadana Vinculante.

En la primera etapa de negociaciones, se optó por trabajar en torno a una sola mesa, intentando evitar la fragmentación de los temas relevantes para la Mesa Social de Aysén. En el camino los mismos actores fueron evaluando que este mecanismo era inviable por el nivel y envergadura de las demandas planteadas en el documento maestro. De esta forma las mesas se convirtieron en el mecanismo más eficiente para abordar cada temática por separado.

El funcionamiento de cada mesa exigía la presencia de un representante del Gobierno Central con poder de decisión directa sobre los temas en debate. En este sentido el criterio fue trabajar de forma paralela, puesto que no todos los actores centrales podrían haber estado al mismo tiempo. Teniendo presente que la división sectorialista afectaría el grado de cohesión de los dirigentes sociales, se concluyó que en todas las mesas estarían los voceros del movimiento para garantizar y firmar los acuerdos sostenidos con el nivel central.

Es importante resaltar que las ocho mesas de negociación desarrollan su respectiva carta de acuerdos firmados entre los dirigentes sociales y la autoridad pública nacional a cargo de estas temáticas. Este proceso se desarrolla en plenitud en la región de Aysén. Posterior al acuerdo sostenido en la Moneda la presencia de autoridades nacionales es masiva y se generan estos documentos que acreditan el compromiso formal del gobierno para con las demandas del Movimiento de Aysén.

Es posible constatar la envergadura de este proceso a través de publicaciones realizadas por blo- gueros del movimiento, que tuvieron por objetivo difundir y controlar todos los procesos que involucraron acuerdos entre las partes. El más destacado de ellos es el blog Despierta Aysén ${ }^{2}$ que publico todas las actas de las mesas firmadas por representantes del gobierno central y dirigentes sociales.

Para profundizar esta revisión, se llevó a cabo un seguimiento de los acuerdos en base a tres categorías de análisis. La primera categoría tiene que ver con investigar acerca de la fuente original del problema público y su respectiva colocación en la agenda pública. En este sentido se tomó como referencia el Plan de Gobierno Regional, carta magna del gabinete regional diseñado a principios del año 2010, configurado en base a las directrices nacionales establecidas por el gobierno de Sebastián Piñera.

La segunda fuente de análisis tiene que ver con el grado de movilización de recursos públicos, donde en primer lugar es valorable reconocer, en el proceso de gestación de política pública, si la iniciativa pasó a ser parte de la programación de inversión pública, lo que no refleja plenamente el real impacto en la gestación de acciones públicas relacionadas con el cumplimiento del compromiso, puesto que tan sólo pudo quedar nominada en la lista de prioridades públicas. Por lo cual se propone revisar si estas iniciativas tuvieron dinámica en la inversión pública a partir de los años en que se gestó la movilización social. Para ello se tomó como referencia de análisis los informes de ejecución presupuestaria establecidos por el Gobierno Regional a partir del Programa Público de Inversión Regional (PROPIR).

Un tercer elemento de impacto en la agenda pública tiene que ver con la valoración subjetiva que le otorgamos como investigadores al grado de relevancia de la acción pública, distinguiendo si la acción pública conllevó un proceso de negociación a nivel legislativo o bien fue un proceso que se desarrolló de manera interna en las carteras ministeriales. En este sentido distinguimos si la iniciativa fue constitutiva en un proyecto de ley o fue una gestión interna al interior de los gabinetes.

Tomando en consideración estas categorías de análisis se procede a presentar los siguientes resultados de la investigación. Para efectos de mejor comprensión del lector iremos desarrollando este análisis según los sectores.

En términos generales la tabla $\mathrm{N}^{\circ} 3$ muestra la distribución de iniciativas comprometidas en los diversas mesas de negociación. Podemos observar que en total se llegaron a comprometer 97 iniciativas,

\begin{tabular}{|c|c|c|c|c|c|}
\hline${ }^{2} \mathrm{Tu}$ & Problema & es & $\mathrm{Mi}$ & Problema & ...Mo- \\
\hline vimiento & Social & por & la & Región de & Aysén \\
\hline
\end{tabular}


siendo en el ámbito de la pesca artesanal la que presenta una mayor presencia de compromisos acordados con $16.49 \%$. Lo secunda el ámbito del agro con la vivienda patagónica con un $12.37 \%$ de las iniciativas.

Como se observa en la tabla $\mathrm{N}^{\circ} 4$, a nivel general, llama la atención que existe un equilibrio en cuanto a las iniciativas según su tipo de solución. Sin embargo, se observa que en el caso de la pesca la mayor parte de las iniciativas tuvieron que ver con el ámbito de gestión. Ahora bien esto se explica puesto que la mayor parte de los compromisos sostenidos en la mesa fueron cambios a la legislación vigente. Ello implica que para dar cobertura a estas iniciativas se movilizaron recursos a nivel legislativo y gubernamental. La relevancia de ello radica en que se hicieron una serie de modificaciones a las resoluciones actuales.

Respecto a las iniciativas de inversión podemos observar una distribución más equitativa en la mayoría de los sectores, destacándose los ámbitos de salud, vivienda, infraestructura (vialidad), fomento, conectividad y educación.

Avanzando en la segunda dimensión de análisis propuesta, respecto al impacto en agenda pública, demostraremos cómo acciones consideradas emblemáticas por los actores sociales tuvieron una importante dinámica de crecimiento en sus caudales financieros.

El proyecto más importante, en cuanto a infraestructura pública, tuvo un incremento desorbitante desde el año 2011 al 2013. El proyecto de normalización del hospital de Puerto Aysén evidencia el cambio más significativo, puesto que si bien era una iniciativa que venía del gobierno anterior, recién el año 2012, después de la movilización social, adquiere la dinámica normal de este tipo de inversión.

De la misma forma es posible observar un incremento significativo en la inversión orientada a educación, especialmente en el ámbito de otorgamiento de becas de apoyo estudiantil que entre el año 2011 y el año 2013 aumenta 35 veces su inversión pública. Cabe destacar además que en este ámbito se crea un programa especial denominado Beca Patagonia Aysén, que si bien logra su aprobación en la ley de presupuesto del año 2012, la revisión del proceso de asignación genera una mayor cobertura para los estudiantes que residen en Aysén.

En la misma lógica de análisis se puede observar un incremento en la ley para el fomento de la inversión privada en obras de riego y drenaje (ley $\mathrm{n}^{\circ}$ 18.450), lo cual tiene directa relación en regularizar derechos de agua que al año 2012 no había tenido solución histórica. Así también el fondo de pesca artesanal que se ve aumentando de manera significativa en la transferencia de recursos a organizaciones del sector pequero artesanal, a partir del año 2013.

Mención especial tiene el reconocido "Bono Leña" que acorde al nivel de inversión estimada (no publicada oficialmente) se estima por datos extraídos en medios de prensa una inversión cercana a los 1.800 millones de pesos en el 2012, beneficiando a un total de 18.000 familias de la región. El año 2013, este bono adquirió mayor relevancia puesto que se aplicó un bono de transferencia directa a las familias de 100.000 pesos beneficiando según las fuentes revisadas a 20.000 familias.

El análisis cambia si nos centramos en los acuerdos comprometidos que no tuvieron resonancia en la gestión del gobierno. Estos acuerdos fueron: Subsidio al transporte e integración física; Mejores condiciones laborales a trabajadores temporeros; Sueldo mínimo regionalizado para los trabajadores del sector privado, cumpliendo el acuerdo de la Mesa Público-Privada; Evaluación de Nivelación de Zona Empleados Públicos; Suspensión administrativa del procedimiento de evaluación de los proyectos Hidroaysén y Río Cuervo; Generación de un mecanismo de participación ciudadana vinculante para la evaluación de los megaproyectos; Canasta básica diferenciada; y Pensión regionalizada para los adultos mayores y personas con capacidades diferentes.

Dichos acuerdos no abordados representan a juicio de varios entrevistados asuntos medulares que motivaron el surgimiento de la movilización social. Destacamos entre ellos los acuerdos de la mesa de participación ciudadana liderada principalmente por dirigentes del Movimiento Patagonia Sin Represas. Asimismo, se puede considerar como emblemático la demanda por mejoras salariales en el sector público, temas que no fueron ni siquiera discutidos en una mesa de negociación.

Existe una brecha importante para hablar de un impacto profundo en la agenda pública. Los avances son realmente asombrosos respecto a la movilización de recursos en cuanto a gestión e inversión y transferencia de subsidios directos hacia los ciudadanos. No obstante, es precario el impacto en la colocación de temas que consiguieran cambiar el rumbo del territorio, otorgándole mayor grado de autonomía y representatividad en la acción pública. 
Incidencia de la agenda social en la agenda pública (El encuentro)

Revisaremos el nivel de incidencia de la ciudadanía en la agenda pública a partir de dos filtros. El primero a partir de los postulados teóricos de Gonzalo Delamaza y Ochsenius (2007), que desarrolla 4 niveles de incidencia y movilización de la ciudadanía en la gestión pública.

Tabla 2: Dimensiones de Incidencia en Política Pública

\begin{tabular}{|l|l|}
\hline Dimensiones & Niveles \\
\hline Incidencia en orientaciones de politica pública. & Nivel 1 \\
\hline Cambios a los métodos de la gestión del gobierno. & Nivel 2 \\
\hline Inclusión y participación de nuevos actores. & Nivel 3 \\
\hline Cultura Organizacional del Estado. & Nivel 4 \\
\hline
\end{tabular}

Fuente: Elaboración propia en base a postulados de Delamaza y Ochsenius (2007)

El segundo filtro aplicado tiene relación con el surgimiento y colocación de la temática en la Agenda pública, utilizando las siguientes definiciones:

- Iniciativa de continuidad: Se comprende como aquella que no tuvo modificación alguna desde cuando fue diseñada en el Plan de Gobierno Aysén 2010-2104.

- Iniciativa reactiva: Se comprende como aquella que existía en el plano de la Agenda Pública previo al movimiento pero que por razones de prioridad política había dejado de estar en la posición para ser ejecutada.

- Iniciativa Emergente: Se comprende como aquella que surge producto de las movilizaciones sociales, ya sea como una solución que emana desde la red de actores sociales o gubernamentales.

A partir de estas definiciones se generaron los cruces respectivos que nos permitieron llegar a la conclusión de que la mayoría de las acciones que se plantean en la agenda pública corresponden al primer nivel de incidencia, donde las acciones son más bien correctivas respecto a las orientaciones de las políticas públicas. Dentro de este primer nivel podemos observar también un equilibrio entre las acciones que emergen producto de la movilización social y acciones que estaban estancadas en la actual gestión gubernamental.

Analizando el segundo nivel de incidencia podemos observar que adquiere mayor relevancia la gestión del gobierno en base a la presión de la movilización social. El número de iniciativas que emerge es mucho mayor que las acciones reactivas. Eso indica en gran medida que la presión social impacta de forma significativa en los métodos de gestión del Gobierno.

Avanzando en este análisis procedemos a revisar en detalle la Creación Mesa Técnica Regional de la pesca artesanal, la creación del Consejo Zonal de Pesca y la Instalación de una Universidad cien por ciento Presencial. Las dos primeras acciones fueron propuestas por el sector pesquero artesanal, para relevar la importancia de analizar desde la región la factibilidad técnica y política de resolución sobre el uso y explotación de los recursos regionales. Por años la definición de estos dos aspectos se zanjaba fuera de la región, donde el factor de proporcionalidad dejaba sin chance a los intereses regionales. Las resoluciones apuntaban a un equilibrio macrosocial entre regiones limítrofes perjudicando la protección y sustentabilidad de la pesca en Aysén y por sobre todo los intereses de los gremios regionales. En términos políticos esta acción puede comprenderse como uno de los actos más determinantes en la búsqueda de autonomía territorial, lo cual se concreta a partir de esta movilización social.

Por último es importante revisar los acuerdos no abordados por la Mesa Social, los cuales dejaron una profunda huella de frustración a escala regional. Se podría eventualmente levantar un sondeo respecto al nivel de éxito de la movilización social desde una visión ciudadana y la sensación generalizada sería que el movimiento social tuvo un alto costo para el nivel de logros alcanzados. La comunidad Aysenina reconoce que fue un hito importante, pero que genero divisiones muy marcadas de adhesión o rechazo.

Esto mismo sucedió con aquellos grupos que eran parte de la Mesa Social como Patagonia Sin Represas, que no lograron concretar un acuerdo fehaciente con su contraparte gubernamental. Por el contrario, a pocos días de conversado el acuerdo con el Subsecretario de Desarrollo Regional, se desarrolló un comité que aprobó la construcción de la Central hidroeléctrica en el Río Cuervo. Por otra parte, se habló de inconstitucionalidad respecto de llevar adelante plebiscitos vinculantes a escala regional.

Más evidente es el caso de la Asociación Nacional de Funcionarios Públicos (ANEF) que no logró siquiera formar una mesa para generar salidas a sus planteamientos. Aún siguen pendientes las exigencias de este gremio para sentarse a dialogar con el gobierno.

En esencia, la no consideración de las iniciativas planteadas, apunta a lo que podríamos entender como una omisión y pérdida de la oportunidad de 
avanzar en un mayor grado de incidencia en política pública. Llama la atención que ninguna de las 97 iniciativas incluidas en este análisis hayan adquirido el nivel 4 de incidencia, donde se apreciaría un mayor grado de impacto en la cultura organizacional del Estado. Este aspecto es relevante puesto que seguirá estando en la banca de las acciones no consideradas y podría sin lugar a dudas ser una nueva motivación de expresión colectiva a escala ciudadana.

\section{Conclusiones y recomendacio- nes}

\section{Aysén: transición de la acción colecti- va a la movilización social}

El movimiento social de Aysén contiene elementos contundentes para constatar que la acción colectiva, avanzó hacia lo que conceptualmente se pude comprender como movimiento social. Este se construye desde una perspectiva histórica, reflejando las diversas identidades que confluyen en la región.

La movilización tuvo como referente inicial al grupo de pescadores de Aysén, los que tradicionalmente luchaban por reivindicaciones particularistas. No obstante, la movilización se expandió cuando este grupo de presión invita a otros organismos que sentían la necesidad de expresar una deuda pendiente con el Estado. Es así como se configura la acción colectiva, convoca a organizaciones de la sociedad civil a movilizarse por varios frentes, que confluían hacia la sensación de abandono por parte del Estado.

Los argumentos de Abalos (1985), nos permiten fortalecer la tesis que este movimiento en su génesis adquiere ribetes de movilización social de carácter reivindicativo a escala territorial, que en suma tiene claros repertorios (Snow y Benford, 1992), pronosticando sus oportunidades de establecer procesos de negociación contra su opositor, que en este caso queda de manifiesto que es el Gobierno de Chile representado por el Ejecutivo de turno.

La identidad de este movimiento se robustece en la medida que logra diferenciarse de su contraparte. La formas de opresión utilizadas por el Ejecutivo, no tan sólo contribuyeron a potenciar la capacidad de movilización, si no también le permitieron aumentar su capacidad de reclutamiento y convocatoria. Todo ello le fue poniendo más presión al proceso de negociación.

La contribución del Movimiento Patagonia Sin
Represas, enriquece la fuerza del movimiento, pone en el tapete temas que en la actualidad se discuten a nivel nacional, y pone en jaque el modelo de crecimiento y explotación de los recursos naturales del país. En reiteradas entrevistas los actores locales asumen que el potencial productivo de Aysén no puede ser discutido por un pequeño grupo de políticos o empresarios. Lo más interesante es que la discusión no se pone en el tema ambiental, sino la discusión contempla el cómo se están tomando decisiones de forma centralizada sin considerar la opinión de los actores del territorio.

En cuanto al terreno o totalidad en el cual estos actores desenvuelven su conflicto y procesos de negociación, sin lugar a dudas que podríamos señalar que la estructura de movilización de actores (Tarrow, 1998) tuvo efectividad hasta que la relación con el Estado (opositor) cambió y se crearon procesos de negociación horizontales.

Cuando once dirigentes del movimiento fueron invitados a la Moneda, se marca un nuevo proceso de movilización de actores, donde muchos de los involucrados sintieron que el movimiento había tranzado su esencia. Para otros era el momento ideal para que lo planteado por el movimiento pase a ser motivo de agenda pública. Sin lugar a dudas que para la opinión pública, este evento marcó políticamente a todo el país, luego de cuarenta días de auto bloqueo, los dirigentes habían logrado ser escuchados y considerados por el nivel central. La reflexión hasta ese momento fue que la región de Aysén había ganado esta lucha. Queda de manifiesto que se genera un espacio de la "policy windows", pasando a ser Aysén como territorio parte de la agenda de gobierno.

\section{Nivel de incidencia del movimiento so- cial de Aysén en la Agenda pública}

Varias de las iniciativas comprometidas en las mesas de trabajo, son parte de negociaciones históricas entre los diferentes grupos de presión o bien como parte de la agenda pública. Uno de los más importantes fue el mundo de la pesca artesanal, principal impulsor de este proceso de movilización, que contaba con una amplia agenda de temas a resolver. La real incidencia en la agenda se manifiesta en la agilización respuestas.

Los agentes del sector público fueron parte de un proceso de apertura de las mismas barreras que el sistema público imponía, para llevar a cabo la agenda regional del gabinete regional. En reiterados casos se mencionó como una oportunidad para el mismo Gabinete, el cual logró colocar sus iniciativas en la priorización del gobierno. Eso contribuyó 
a que la agenda pública a escala regional tuviera mejores resultados.

En el escenario político, los actores más desprotegidos y debilitados fueron los agentes regionales, quienes perdieron fuerza y legitimidad ante los actores locales, producto de esta deslegitimación e incapacidad para resolver temas históricos que estaban en la agenda, y que producto de esta movilización logran destrabarse.

Hay otros temas a nivel transversal que se reconocen como incumplidos, y que paradójicamente fueron los que movilizaron a una gran cantidad de actores. Este es el caso de los funcionarios públicos y Patagonia Sin Represas.

Sidney Tarrow (1998), nos ilustraba acerca de los riesgos que conllevan las acciones colectivas que no fortalecen su estructura de movilización. Puesto que esto podría desencadenar la desarticulación de los actores, pasando de luchas por reivindicaciones colectivas a lucha soluciones particularistas, incluso clientelares.

Una arista no medible cuantitativamente es la capacidad política del movimiento para sostener sus demandas y convertirlas en oportunidad para el colectivo. La incorporación de los líderes sociales al proceso electoral cuestionó el involucramiento con la identidad y la lucha del movimiento.

En las entrevistas es posible reconocer que este evento social transformó el modo de hacer gestión pública. Los entrevistados del ámbito público reconocen el aprendizaje y la falta de oportunidades que existían para generar acuerdos consensuados con la comunidad. Este aprendizaje se traslada a la nueva forma de establecer acuerdos construyendo las soluciones a las demandas con la ciudadanía. Paradójicamente, el reflejo de ello ha permitido que la gestión de algunas acciones en base a las demandas del movimiento, se evalúen comunitariamente como el único logro del movimiento, reconociendo en el bono de leña esta nueva gestión pública.

Esto se fue facilitando puesto que hoy la sensibilidad del nivel central con Aysén permite avanzar en soluciones que están a la mano de la gestión. Un logro transversal de esta movilización fue visibilizar a Aysén como un territorio que requiere respuestas a sus inquietudes. Eso puede ser parte del proceso de presión que obliga al nivel central a priorizar la agenda por Aysén, o bien podría ser que las manifestaciones contribuyeron a reconocer las particularidades de este territorio.

El escenario político de movilización dio paso al proceso de negociación que dejó ganadores y perdedores. Actualmente estamos viviendo otro proceso de incidencia de ciudadanía, en donde la comunidad movilizada le traspasa poder a sus líderes, depositando sus esperanzas de cambio político.

Gran parte de lo que pudo ser realizado a nivel de gestión nacional y que se planteó en el movimiento fue agilizado y comprometido, pero gran parte de aquello que requería de capacidades territoriales y soluciones pertinentes a la realidad regional no fue asumido ni siquiera como compromiso. Los plebiscitos vinculantes para determinar la implementación de megaproyectos, el sueldo regionalizado, la conectividad física principalmente.

Todo ello hubiera sido más potente si se hubiera demostrado que el movimiento también impactó en las estructuras del Estado, logrando nuevos procesos en la gestión pública que traspasen más poder y autonomía a los territorios. El opositor se hizo más fuerte puesto que ganó aliados que pasaron a ser parte de la elite gobernante.

Se percibe del proceso de movilización en Aysén, el asentamiento de las bases para la germinación de la legitimidad invisible (Rosanvallon, 2009), esa que es capaz de poner en jaque al "establishment", que utiliza mecanismos electorales para potenciar la autonomía y la democracia. Además se moviliza ante las injusticias sociales.

\section{Recomendaciones y sugerencias}

Los hallazgos de la investigación van dando cuenta de la amplia brecha que existe actualmente entre el diseñador de políticas públicas y el implementador de políticas públicas. Asunto que es ampliamente abordado por Waissbluth (2007), en su artículo sobre el déficit de análisis y articulación entre el diseño e implementación. Dicha brecha se profundiza si analizamos con atención que el proceso de hechura de políticas públicas carece del enfoque territorial. Cuestión que se ha agudizado con el alzamiento de comunidades empoderadas, exigiendo que la gestión pública considere su diversidad. Y no tan sólo que la considere, si no que la incorpore en su cultura organizacional.

Se sugiere incorporar el elemento "T" en la hechura de políticas públicas. Hacemos esta analogía señalando la "T" de "Territorio" en relación a lo que Waissbluth (2007) utiliza como "I" para referirse a "Implementación". El sistema actualmente bajo ninguna perspectiva está incorporando categorías de análisis que les permita configurar un tipo de política pública que responda a las especificidades de cada espacio. La complejidad de aquello no pasa tan sólo por generar un andamiaje de gestión pública capaz de razonar para los territorios, sino 
más bien desde y para los territorios.

La necesidad de implementar este enfoque en el proceso de hechura de políticas públicas requiere de urgencia. La suma de poderes locales va incrementándose y el empoderamiento de la comunidad va buscando mecanismos no institucionalizados para expresar sus intereses. La estructura actual no está preparada para resolver el incremento de conflictos sociales a escala territorial, lo cual podría traducirse en períodos de inestabilidad política. Por esta razón debiéramos poner en marcha procesos que permitan potenciar el andamiaje estructural de los territorios.

Tomando varias de las propuestas que se han mencionado en esta materia, reconocemos la relevancia de crear centros especializados de información para la formulación de políticas públicas desde y para los territorios. A la vez se debiera fortalecer la autonomía de los Gobiernos Regionales en esta materia, generando las sustentabilidad de los instrumentos de planificación regional. Al mismo tiempo las Unidades de Planificación Regional debieran tener mayor atribución de fiscalización de las políticas públicas sectoriales, enmendando el rumbo de instrumentos que no representan las lógicas territoriales.

Finalmente se debieran ampliar mecanismos de trabajo con la sociedad civil para la formulación de políticas públicas. Quebrando el esquema actual donde la comunidad es considerada un hito para validar lo que decide el gestor de política pública, abriendo paso a la generación de políticas públicas co-construidas donde el nivel de incidencia de la comunidad apunte al nivel de cambio en la cultura organizacional del Estado.

\section{Referencias}

Abalos, J. (1985). Introducción al estudio de los movimientos regionales. El trimestre Económico, $206 .: 379-410$.

Aguilar, L. F. (2007). Problemas públicos y agenda de gobierno.

Boisier, S. (2004). La doctrina (oculta) de la descentralización chilena. Ministerio de Planificación y
Cooperación de Chile, MIDEPLAN, Desarrollo Regional.

Borgés, R. (1995). El Estudio de Caso Como Instrumento Pedagógico y de Investigación en Políticas Públicas. Facultad de Ciencias Físicas y Matemáticas, Departamento de Ingeniería Industrial, Universidad de Chile.

Delamaza, G. y Ochsenius, C. (2007). Innovaciones en los vínculos entre sociedad civil y Estado en Chile: Su impacto en la gobernanza democrática. Documento de Trabajo, 19.

Kingdon, J. W. (1984). Agendas, alternatives, and public policies. Boston: Little, Brown.

Lira Cossio, L. (2003). La cuestión regional y local en América Latina. CEPAL - SERIE Gestión pública, 44.

Subsecretaria de Desarrollo Regional (2011a). Estudio identificación de territorios aislados 2011. División de Políticas y Estudios Departamento de Estudios y Evaluación Unidad de Análisis Territorial.

Subsecretaria de Desarrollo Regional (2011b). Informe final metodología para la definición de territorios especiales Valparaíso.

Tricot, T. (2011). Sociedad, Estado-Nación, Sujeto y Movimientos Sociales. Revista de Psicología Universidad Viña del Mar, 1:128-147.

Universidad Central de Chile (2012). (2012). Diagnóstico y Línea de Base de las Regiones de Chile en los Ámbitos Económico, Social y Territorial-Ambiental.

Waissbluth, M. (2007). Déficit de Vitamina "I": Las omisiones de implementación de las políticas públicas o bien: la economía política de la ineficiencia pública. Ingeniería Industrial, Universidad de Chile.

Yáñez, B. y Amigo, G. (2009). La reforma administrativa como reacción a una crisis política. el gobierno de ricardo lagos (2000-2006). TékhneRevista de Estudos Politécnicos, 11:91-125.

Zibechi, R. (2003). Los movimientos sociales latinoamericanos: tendencias y desafíos. Observatorio Social de América Latina, 9. 